\title{
Técnica de "slalom" quirúrgico en estenosis de canal lumbar multinivel. Serie de casos tratados de manera simultánea con descompresión endoscópica y tubular con asistencia microscópica bilateral
}

\author{
Nicolás Coombes, Eduardo Galaretto, Juan Pablo Guyot, Norberto Fernández, Cristian Fuster \\ Axial Grupo Médico, Ciudad Autónoma de Buenos Aires, Argentina
}

\begin{abstract}
RESUMEN
Introducción: La laminectomía unilateral para descompresión bilateral por abordaje único ha tomado relevancia en pacientes con estenosis multinivel. Cuando se realiza en más de un nivel por un abordaje único, de manera alterna y de forma cruzada, al abordaje anterior es conocida como técnica de "slalom". El objetivo de este artículo es presentar una serie de casos tratados con la técnica de "slalom" con asistencia endoscópica y microscópica bilateral, simultánea, en pacientes con estenosis de canal lumbar multinivel. Materiales y Métodos: Análisis retrospectivo de pacientes tratados de forma simultánea, entre enero de 2017 y enero de 2018, todos operados por el mismo equipo quirúrgico con separadores tubulares, y asistencia endoscópica y microscópica simultánea. Resultados: Se incluyó a 4 hombres, con una edad promedio de 73.5 años y patología lumbar multinivel. Se descomprimieron 10 segmentos (2,5 media de niveles por paciente), con un tiempo quirúrgico promedio de 107 minutos. No hubo complicaciones asociadas y los pacientes recibieron el alta hospitalaria el día de la cirugía. Conclusiones: La técnica de "slalom" mínimamente invasiva resulta ser un procedimiento muy eficaz para resolver síntomas de estenosis multinivel asociada a una técnica combinada bilateral con dos equipos quirúrgicos para el tratamiento de este tipo de pacientes.
\end{abstract}

Palabras clave: Canal lumbar estrecho; descompresión cruzada; "slalom" quirúrgico; descompresión lumbar mínimamente invasiva.

Nivel de Evidencia: IV

Slalom Technique as a Treatment in Multilevel Lumbar Spinal Stenosis. Series of Cases Treated
Simultaneously with Endoscopic and Tubular Decompression with Microscopic Assistance

\section{ABSTRACT}

Introduction: Unilateral laminectomy for bilateral decompression (ULBD) by single approach has become relevant in patients with multilevel stenosis. When it is performed at more than one level with a single approach, alternately and crosswise to the previous approach, it is known as a "slalom" technique.The objective of this article is to present a series of cases treated with the slalom technique with simultaneous bilateral endoscopic and microscopic assistance in patients with multilevel lumbar canal stenosis. Materials and Methods: Retrospective analysis of patients treated simultaneously from January 2017 to January 2018, all operated by the same surgical team with tubular separators and simultaneous endoscopic and microscopic assistance. Results: Four patients, all male, with an average age of 73.5 years with multilevel lumbar pathology, were included. In total, 10 segments were decompressed (2.5 levels per patient, on average), with an average surgical time of 107 minutes. There were no associated complications, the patients were discharged within the day of surgery. Conclusions: The minimally invasive Slalom technique is a very effective procedure to resolve symptoms of multilevel stenosis associated with a bilateral combined technique with two surgical teams, resulting in a viable option for the treatment of this type of patient.

Key words: Lumbar spinal stenosis; over the top decompression; surgical slalom; minimally invasive lumbar decompression. Level of Evidence: IV

Recibido el 17-8-2019. Aceptado luego de la evaluación el 6-9-2020 • Dr. NICOLÁS COOMBES • nicolascoombes@gmail.com

Cómo citar este artículo: Coombes N, Galaretto E, Guyot JP, Fernández N, Fuster C. Técnica de "slalom" quirúrgico en estenosis de canal lumbar multinivel. Serie de casos tratados de manera simultánea con descompresión endoscópica y tubular con asistencia microscópica bilateral. Rev Asoc Argent Ortop Traumatol 2021;86(2):175-184. https://doi.org/10.15417/issn.1852-7434.2021.86.2.1013 


\section{INTRODUCCIÓN}

A medida que aumenta el promedio de edad de la población, se incrementa la cantidad de pacientes que sufre una enfermedad degenerativa dolorosa de la columna lumbar. ${ }^{1,2}$ Tradicionalmente se ha considerado a la laminectomía como el tratamiento de elección para pacientes con estenosis de canal espinal que no responde al tratamiento conservador. ${ }^{3}$ La descompresión aislada abierta puede causar inestabilidad y dolor lumbar recurrente, y terminar en una cirugía de fusión. ${ }^{4,5}$

En los últimos años, se han desarrollado alternativas menos invasivas a la laminectomía. ${ }^{6}$

La laminotomía unilateral para descompresión bilateral, en particular, se considera una técnica eficaz, segura y que reduce el daño tisular al compararla con las técnicas abiertas. ${ }^{7-10}$

La cirugía de descompresión lumbar llamada "descompresión cruzada" se realiza a través de un abordaje unilateral y permite lograr una descompresión de forma bilateral. Cuando esta técnica se efectúa en más de un segmento con abordajes alternos se denomina técnica de "slalom" y se ha convertido en una opción de tratamiento quirúrgico para la estenosis espinal lumbar degenerativa multinivel. ${ }^{11}$

En las cirugías abiertas, la laminectomía se asocia a incisiones cutáneas más largas para alcanzar dos o más segmentos. Los músculos se deben retraer más y la resección parcial de las facetas inferior y superior se debe realizar en dos o más segmentos en el mismo lado del abordaje. Esto produce un mayor daño colateral unilateral para los músculos y las articulaciones, lo que contrarresta la filosofía microquirúrgica de esta técnica. ${ }^{12,13}$

El objetivo de este artículo es proporcionar una descripción técnica de la descompresión lumbar a varios niveles, de forma alternada, tipo "slalom", simultánea con dos cirujanos, y comunicar los resultados preliminares.

\section{MATERIALES Y MÉTODOS}

Se llevó a cabo un análisis retrospectivo de pacientes que se sometieron a descompresión mínimamente invasiva con técnica de "slalom" multinivel por estenosis de canal lumbar entre enero de 2017 y enero de 2018.

Se evaluaron el tiempo quirúrgico, los niveles tratados, las complicaciones operatorias, la estancia hospitalaria. Todos los pacientes fueron evaluados antes de la cirugía, a los 30 días y al año de la operación mediante la escala analógica visual y la satisfacción del paciente se determinó usando la escala de Weiner y los criterios de Macnab modificados.

Los cuatro pacientes de la serie tenían estenosis de canal lumbar multinivel en las imágenes por resonancia magnética (Figura 1) y dolor radicular de larga data, y claudicación en la marcha sin inestabilidad segmentaria, que fueron evaluados en las radiografías en flexión y extensión.

En esta serie, los diagnósticos fueron: discopatía degenerativa multinivel (3 pacientes), estenosis congénita de canal lumbar (un caso), canal lumbar estrecho por hipertrofia del ligamento amarillo (4 pacientes).

Los pacientes fueron tratados por el mismo equipo quirúrgico, de forma simultánea. Se utilizó la prueba t de Student para las variables apareadas independientes preoperatorias y posoperatorias para obtener su valor estadístico. Se empleó el programa EPI Info 7.2.2.6 (2018). Se indicó cirugía a todos los pacientes en forma programada debido a una respuesta parcial a los tratamientos conservadores.

\section{Téenica quirúrgica}

Se llevó a cabo, de manera simultánea, con un cirujano por lado. Uno de ellos realizó la cirugía tubular con asistencia endoscópica (equipo EasyGo 1, Karl-Storz), mientras que, del lado contralateral, se utilizó un microscopio OPMI Pentero 800 (Zeiz) con separador tubular METRX (Medtronic).

Se colocó al paciente en decúbito prono y se le administró anestesia general. Se utilizó radioscopia lateral para marcar la localización de la incisión cutánea. Ambos cirujanos establecen las incisiones de acuerdo con el nivel por tratar (Figura 2).

Ambos procedimientos comparten el mismo sistema de ingreso por lo que se usan dilatadores y canal de trabajo de $20 \mathrm{~mm}$ (Figura 3).

A fin de poder realizar el trabajo de manera simultánea, se realizan descompresiones en niveles alternos y con el microscopio ligeramente angulado para no entorpecer los movimientos del cirujano enfrentado. Esta disposición en el quirófano con el uso de la asistencia endoscópica proporciona la ventaja técnica de permitir el uso de ambos equipos al mismo tiempo y no dificultar las maniobras del cirujano de enfrente, porque la óptica es de $25^{\circ}$. Se efectúa la descompresión según la técnica de laminotomía unilateral para descompresión bilateral descrita por Hartl y cols. ${ }^{1}$ El objetivo es realizar una flavectomía completa desde el pedículo caudal hasta la inserción craneal del ligamento amarillo. 

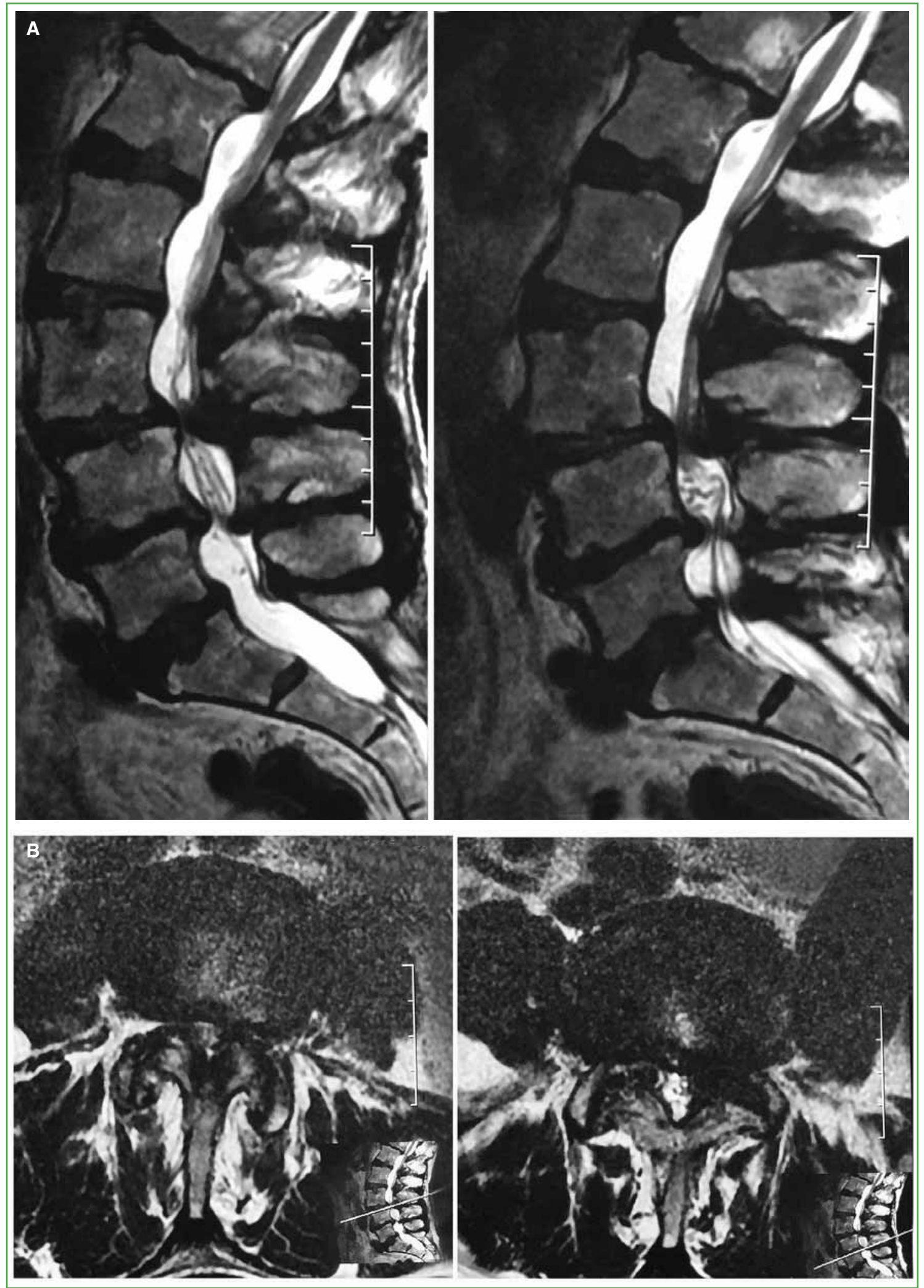

Figura 1. Resonancia magnética en secuencia T2. A. Cortes axiales. Se visualiza la estenosis de canal a varios niveles. B. Cortes sagitales. Se observa el compromiso discal multinivel. 


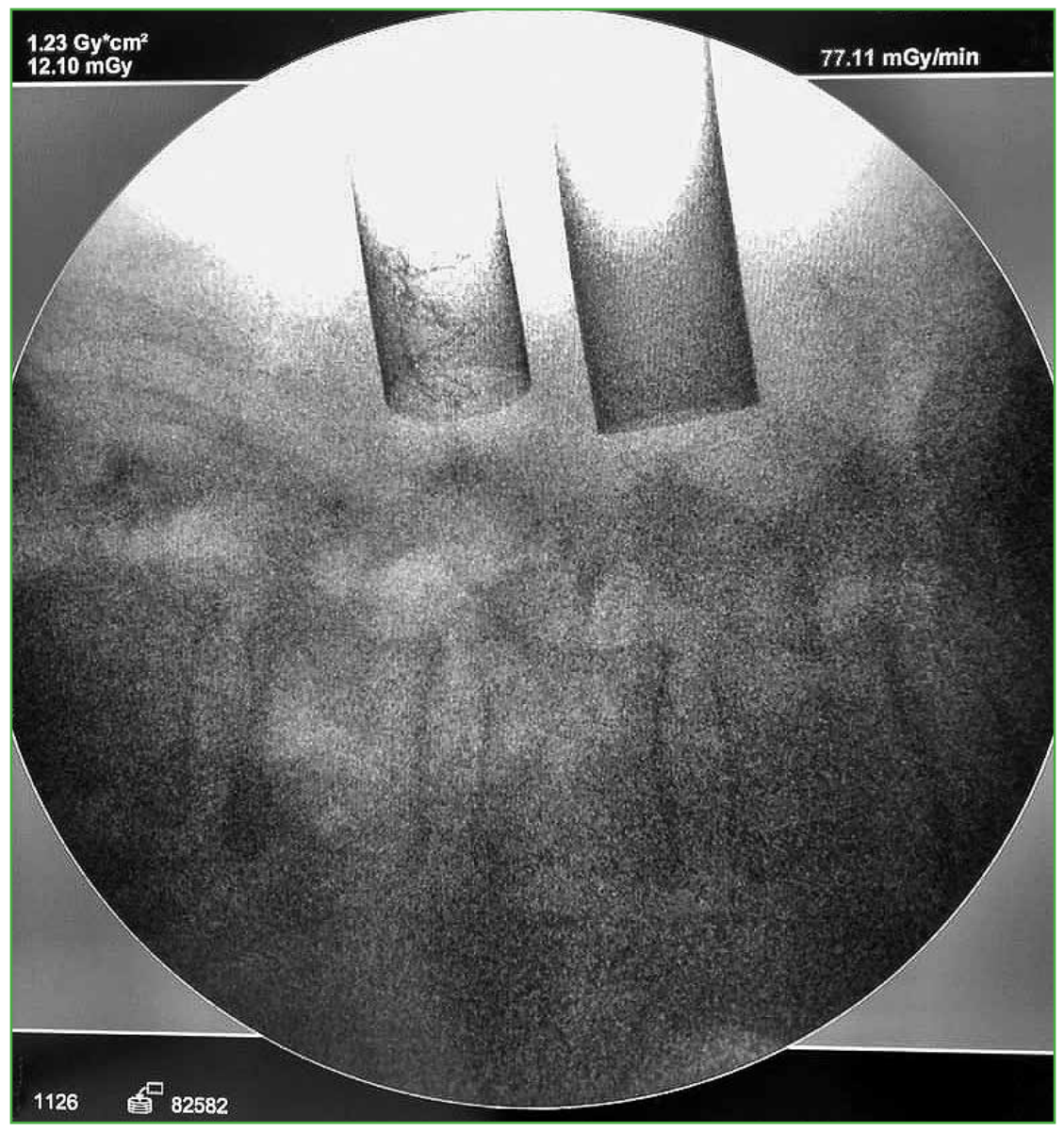

Figura 2. Posición de ambos tubos, vista lateral del intensificador de imágenes.

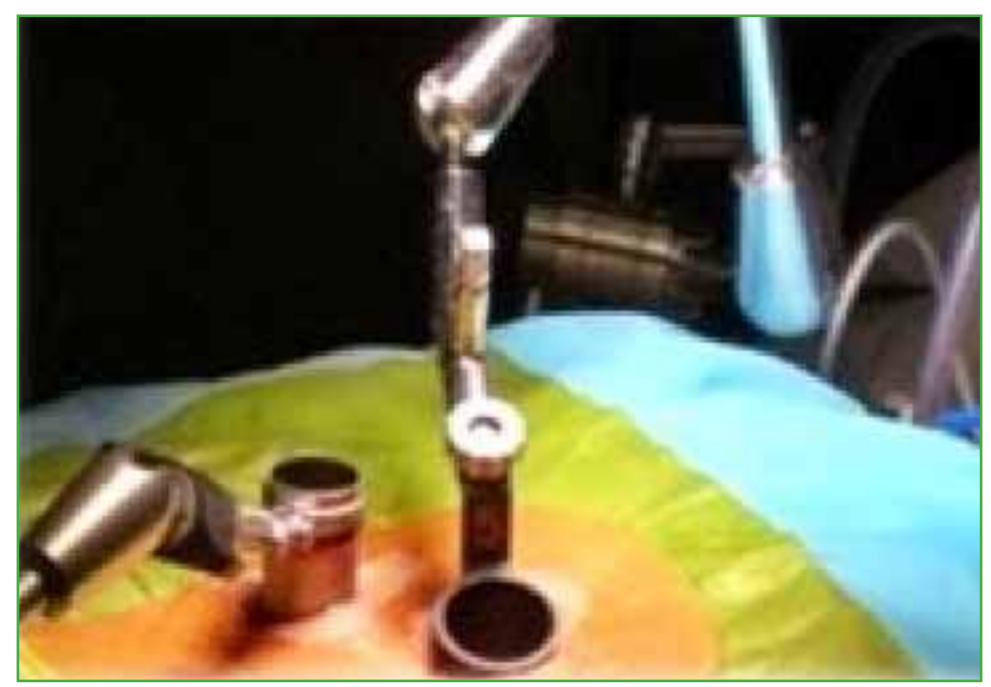

Figura 3. Disposición quirúrgica de ambos sistemas. 
La secuencia es distinta para ambos lados, podríamos definirlo como primera etapa tomando como referencia al operador del lado que utiliza el microscopio y realiza la descompresión homolateral al abordaje y, en el lado contralateral, donde se usa el endoscopio que se sitúa en el nivel supra-adyacente o infra-adyacente; en este, la cirugía se inicia por la descompresión cruzada al lado del abordaje utilizado mediante la técnica over the top (Figura 4).

En una segunda etapa, tomando como referencia la secuencia del operador que utiliza el microscopio, se inclina la mesa de operaciones junto con un ángulo del tubo hacia la línea media para realizar la descompresión cruzada y, del lado del endoscopio, se descomprime el lado homolateral al abordaje (Figura 5).

Antes de retirar el tubo, se realiza un lavado profuso del lecho con abundante solución fisiológica. Hemostasia de los planos venosos epidurales. Cierre hermético de la fascia, el tejido celular subcutáneo y la piel.

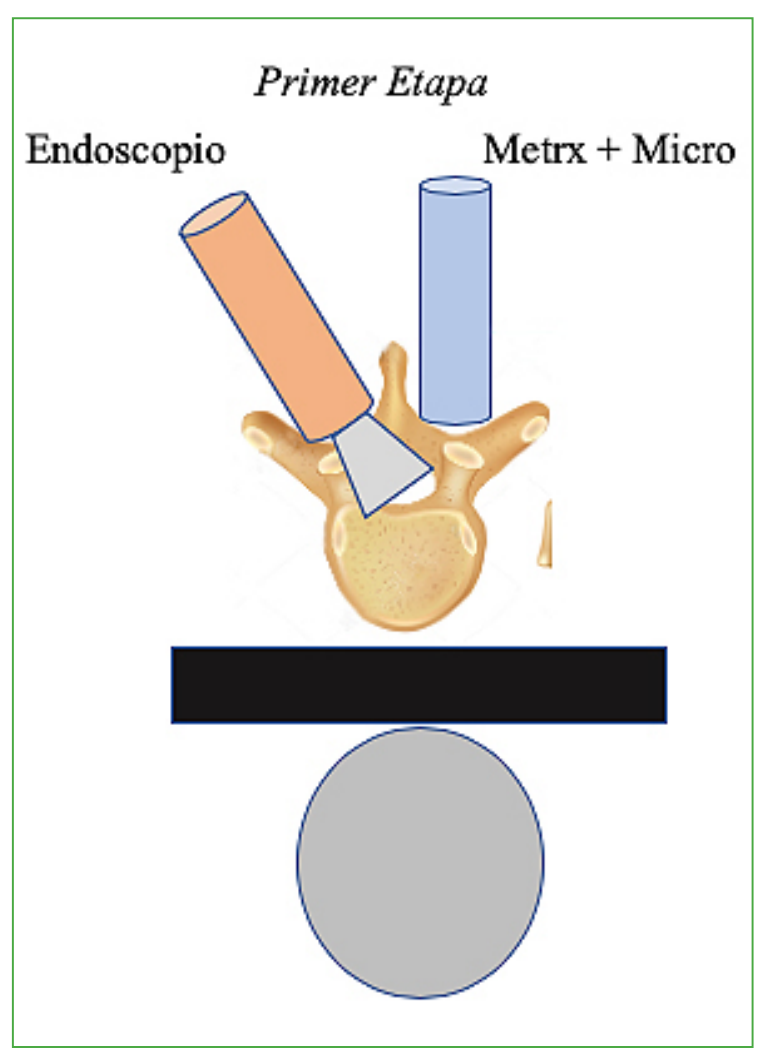

Figura 4. Primera etapa de la cirugía tomando como referencia al cirujano que utiliza el microscopio. Comienza descomprimiendo el lado homolateral al abordaje. El endoscopio se usa para descomprimir el contralateral.

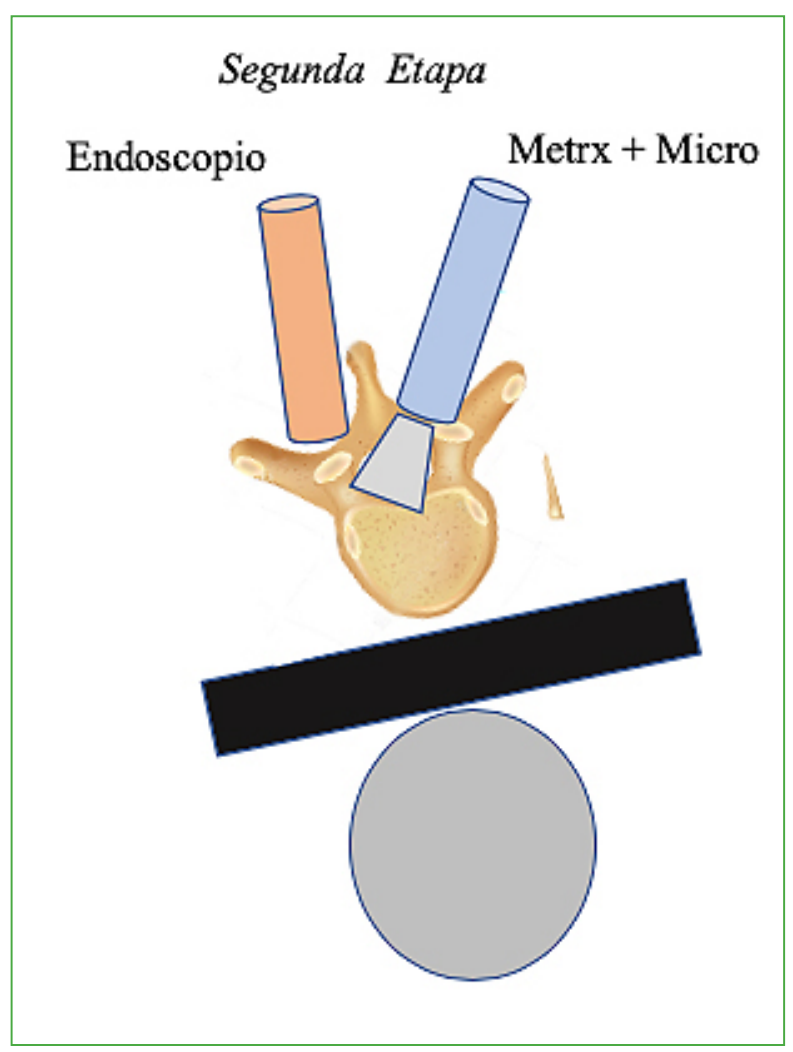

Figura 5. Segunda etapa de la cirugía. Se inclina la mesa de operaciones para lograr la descompresión contralateral con el microscopio. El endoscopio se usa para la descompresión en el mismo lado del abordaje.

\section{RESULTADOS}

Entre enero de 2017 y enero de 2018, cuatro hombres (edad promedio 73.5 años, rango 70-77) se sometieron a cirugía. Las comorbilidades eran 13: hipertensión arterial sistémica (4 casos), anticoagulación por vía oral (3 casos), enfermedad pulmonar obstructiva crónica (2 casos), coronariopatía/colocación de una endoprótesis (3 casos), enfermedad de Parkinson (1 caso). La decisión sobre en qué lado se realizaría cada una de las técnicas se tomó según la comodidad del equipo (Tabla 1). El total de niveles descomprimidos fue 10 (media de 2,5 niveles por paciente) con afectación entre L2 y L5. Los segmentos más comprometidos fueron L3 y L4 en el $70 \%$ de los casos, seguidos de L5 y L2. El tiempo promedio de cirugía fue de 107,75 min (rango 86-123) y no se registraron complicaciones durante el procedimiento. 
Tabla 1. Datos demográficos

\begin{tabular}{|l|c|}
\hline Número de pacientes & 4 \\
\hline Sexo & Masculino \\
\hline Edad & $73.5(70-77)$ \\
\hline Bloqueos previos & 51 \\
\hline Comorbilidades & 13 \\
\hline Hipertensión arterial & 4 \\
\hline Anticoagulación oral & 3 \\
\hline Enfermedad pulmonar obstructiva crónica & 2 \\
\hline Coronariopatías & 3 \\
\hline Enfermedad de Parkinson & 1 \\
\hline
\end{tabular}

En el posoperatorio inmediato, se les indicó a los pacientes la deambulación precoz a partir de las $2 \mathrm{~h}$ de finalizada la cirugía. Todos fueron dados de alta con un tiempo de internación promedio de 11.5 h (rango 9-18). No se registraron complicaciones posoperatorias inmediatas ni en el seguimiento que, en promedio, se prolongó 26 meses (rango 18-33) (Tabla 2).

Utilizando los criterios de satisfacción de Macnab modificados, tres pacientes consideraron el resultado como excelente y uno, como muy bueno. Según la escala de Weiner, los cuatro pacientes percibían que el procedimiento había sido muy o bastante exitoso y lo recomendarían a otros pacientes.

La mejora de los resultados clínicos obtenidos fue muy favorable, el puntaje promedio de la escala analógica visual fue de 8/10; en el posoperatorio inmediato fue de $2 / 10(\mathrm{p}<0,05)$ y $2,2 / 10$ al año.

Tabla 2. Datos quirúrgicos y niveles tratados

\begin{tabular}{|l|c|}
\hline Tiempo & 107,7 (86-123) \\
\hline Estancia hospitalaria & $11,5 \mathrm{~h}(9-18 \mathrm{~h})$ \\
\hline Complicaciones & No \\
\hline Reoperaciones & No \\
\hline Niveles afectados & 10 \\
\hline L2-L3 & 1 \\
\hline L3-L4 & 4 \\
\hline L4-L5 & 4 \\
\hline L5-S1 & 1 \\
\hline
\end{tabular}


En la Figura 6 y la Tabla 3, se resumen la valoración clínica posoperatoria mediante la escala analógica visual y la satisfacción según los criterios de Weiner y Macnab modificados.

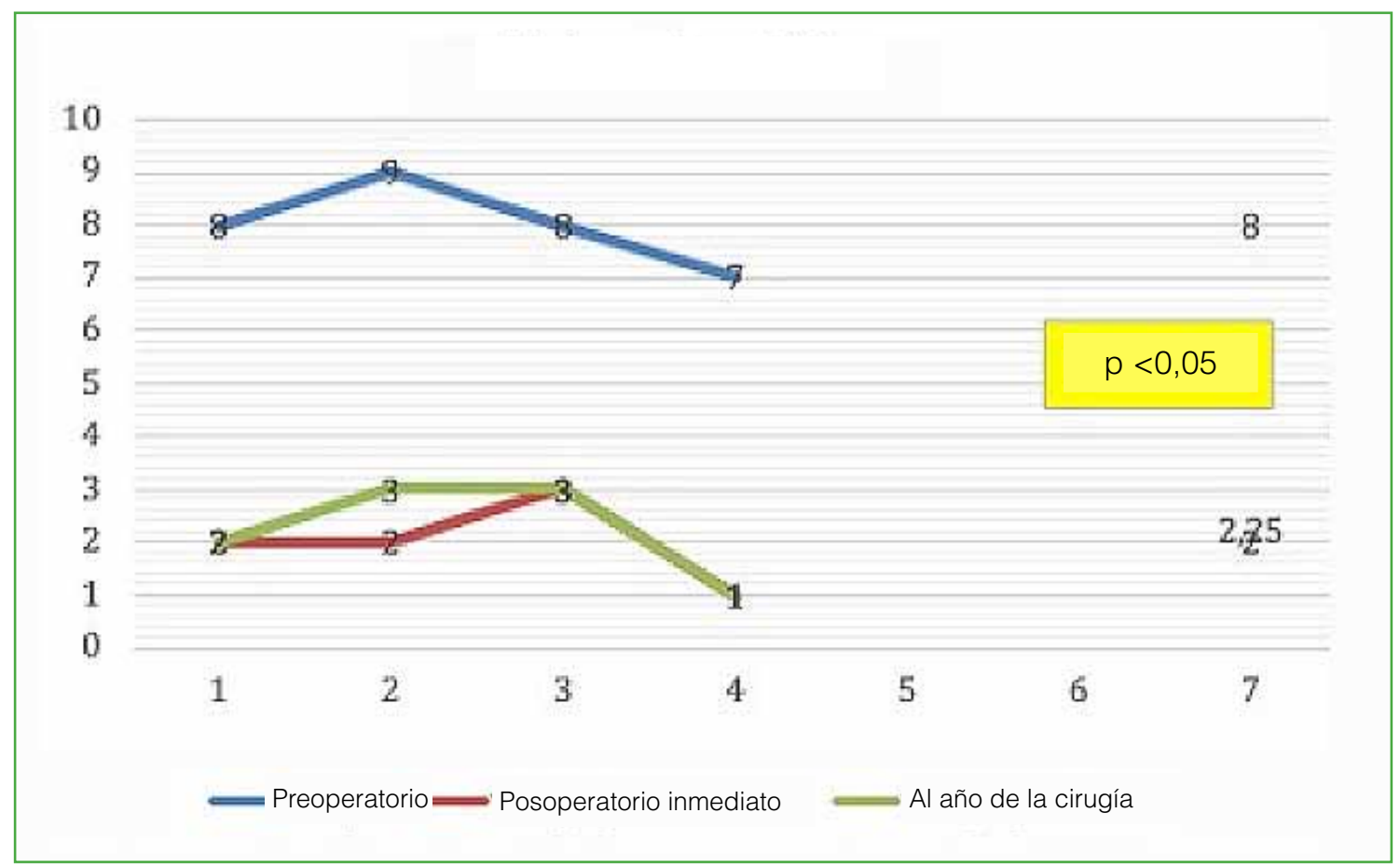

Figura 6. Escala analógica visual en el posoperatorio inmediato y al año de la cirugía con una mejoría significativa $(\mathrm{p}<0,05)$.

Tabla 3. Resultados de satisfacción posoperatoria según los criterios de Macnab modificados y de Weiner

\begin{tabular}{|l|l|}
\hline Satisfacción posoperatoria & \\
\hline Macnab modificado & $3 / 4$ \\
\hline Excelente & $1 / 3$ \\
\hline Bueno & \\
\hline Regular & \\
\hline Malo & \\
\hline Weiner & \\
\hline Éxito & $2 / 4$ \\
\hline Bastante & $2 / 4$ \\
\hline No mucho & \\
\hline Fracaso & \\
\hline
\end{tabular}




\section{DISCUSIÓN}

El avance progresivo en la expectativa y la calidad de vida sumado a la posibilidad de contar con mejores opciones diagnósticas han hecho de la estenosis espinal la patología más frecuente en los centros de columna vertebral del mundo occidental. ${ }^{4,8}$ La laminectomía convencional ha sido el tratamiento quirúrgico tradicional durante décadas. Aunque el desarrollo posoperatorio de inestabilidad segmentaria es un problema multifactorial, el daño innecesario a las estructuras anatómicas que estabilizan al complejo capsuloligamentario siempre ha sido un problema con esta técnica. ${ }^{14} \mathrm{Al}$ mismo tiempo, con esta estrategia de cirugía, aumenta la posibilidad de formación de tejido cicatricial y fibrosis epidural con la potencial aparición de síntomas radiculares en el posoperatorio. ${ }^{12,13}$

La descompresión cruzada mediante un abordaje unilateral tiene ciertas ventajas: reduce, al mínimo, significativamente los riesgos de fibrosis epidural, los músculos se retraen solo en un lado y el área del canal espinal que está expuesta al tejido circundante permanece pequeña. Esto reduce el área de formación potencial de cicatrices. Además, la integridad de la articulación facetaria contralateral permanece casi completamente intacta. ${ }^{11}$ Estas ventajas, por lo general, se pierden en pacientes con enfermedades multisegmentarias que representan más del $50 \%$ de la población en personas $>65$ años. ${ }^{15}$

Al mismo tiempo, en pacientes con patología en múltiples segmentos, realizar un abordaje unilateral ingresando siempre del mismo lado conduce a un aumento de la lesión muscular, se agrega además que la eliminación de la parte medial de la faceta descendente en dos o más niveles de un mismo lado puede provocar problemas funcionales unilaterales. Esto adquiere aún más importancia cuando la estenosis espinal se asocia con una deformidad degenerativa, como la espondilolistesis degenerativa o la escoliosis de novo. ${ }^{16,17}$

La técnica de "slalom" podría ser una opción para reducir, al mínimo, el daño colateral o hacerlo más equilibrado manteniendo las ventajas de la cirugía mínimamente invasiva en pacientes seleccionados. Si bien se trata de una serie pequeña de pacientes, no se produjeron complicaciones.

En la mayoría de los reportes, se describe la técnica tratando cada segmento en forma diferida. Recientemente se ha publicado el resultado del tratamiento en tándem de múltiples segmentos con asistencia microscópica bilateral y los resultados funcionales fueron similares a los de nuestra serie. ${ }^{18}$

No hemos encontrado reportes sobre el tratamiento con técnicas combinadas simultáneas para este cuadro. En nuestro caso, el uso de dos métodos diferentes (endoscopio y microscopio) obedece a curvas de aprendizaje diferentes, pero principalmente a un mejor uso del espacio físico del quirófano.

\section{Limitaciones y ventajas}

Esta serie expresa los primeros resultados de nuestra experiencia con una técnica nueva. Es probable que el tiempo quirúrgico disminuya con una curva de aprendizaje creciente. ${ }^{19}$ El objetivo era presentar una nota técnica de cómo planeamos el tratamiento en este tipo de enfermedades. Consideramos una ventaja poder utilizar esta técnica en pacientes con múltiples comorbilidades para evitar el ingreso en el quirófano en etapas.

Una limitación y un desafío obvio de esta técnica es la mayor necesidad de recursos, ya que requiere de acceso a un endoscopio y su curva de aprendizaje. En nuestro caso, lo utilizamos por no contar con acceso a otro microscopio y el espacio reducido dentro del quirófano.

\section{CONCLUSIONES}

Los resultados resumen nuestra primera experiencia con las técnicas combinadas simultáneas para la descompresión lumbar mínimamente invasiva multinivel. Esta técnica ha sido segura y permite obtener muy buenos resultados. Podría considerarse como una opción válida para tratar pacientes con estenosis de canal lumbar multinivel y múltiples comorbilidades, por ser una técnica eficaz para aliviar los síntomas y que, al ser efectuada de manera combinada con dos equipos, evitaría la necesidad de múltiples intervenciones. 
Conflicto de intereses: Los autores no declaran conflictos de intereses.

ORCID de E. Galaretto: https://orcid.org/0000-0003-4121-8228 ORCID de J. P. Guyot: https://orcid.org/0000-0001-6524-4426
ORCID de N. Fernández: https://orcid.org/0000-0002-5250-5399 ORCID de C. Fuster: https://orcid.org/0000-0002-5293-0846

\section{BIBLIOGRAFÍA}

1. Costa F, Sassi M, Cardia A, Ortolina A, De Santis A, Luccarell G, et al. Degenerative lumbar spinal stenosis: analysis of results in a series of 374 patients treated with unilateral laminotomy for bilateral microdecompression. $J$ Neurosurg Spine 2007;7(6):579-86. https://doi.org/10.3171/SPI-07/12/579

2. Deyo RA, Ciol MA, Cherkin DC, Loeser JD, Bigos SJ. Lumbar spinal fusion. A cohort study of complications, reoperations, and resource use in the Medicare population. Spine (Phila Pa 1976) 1993;18(11):1463-70. PMID: 8235817

3. Becker P, Bretschneider W, Tuschel A, Ogon M. Life quality after instrumented lumbar fusion in the elderly. Spine (Phila 1976) 2010;35(15):1478-81. https://doi.org/10.1097/BRS.0b013e3181c62294

4. Fredman B, Arinzon Z, Zohar E, Shabat S, Jedeikin R, Fidelman ZG, et al. Observations on the safety and efficacy of surgical decompression for lumbar spinal stenosis in geriatric patients. Eur Spine J 2002;11(6):571-4. https://doi.org/10.1007/s00586-002-0409-7

5. Kilinçer C, Steinmetz MP, Sohn MJ, Benzel EC, Bingaman W. Effects of age on the perioperative characteristics and short-term outcome of posterior lumbar fusion surgery. J Neurosurg Spine 2005;3(1):34-9. https://doi.org/10.3171/spi.2005.3.1.0034

6. Machado GC, Ferreira PH, Yoo RI, Harris IA, Pinheiro MB, Koes BW, et al. Surgical options for lumbar spinal stenosis. Cochrane Database Syst 2016;11(11):CD012421. https://doi.org/10.1002/14651858.CD012421

7. Overdevest GM, Jacobs W, Vleggeert-Lankamp C, Thome C, Gunzburg R, Peul W. Effectiveness of posterior decompression techniques compared with conventional laminectomy for lumbar stenosis. Cochrane Database Syst Rev 2015;(3):CD010036. https://doi.org/10.1002/14651858.CD010036.pub2

8. Oertel MF, Ryang YM, Korinth MC, Gilsbach JM, Rohde V. Long-term results of microsurgical treatment of lumbar spinal stenosis by unilateral laminotomy for bilateral decompression. Neurosurgery 2006;59(6):1264-70. https://doi.org/10.1227/01.NEU.0000245616.32226.58

9. Schöller K, Alimi M, Cong GT, Christos P, Härtl R. Lumbar spinal stenosis associated with degenerative lumbar spondylolisthesis: a systematic review and meta-analysis of secondary fusion rates following open vs. minimally invasive decompression. Neurosurgery 2017;80:355-67. https://doi.org/10.1093/neuros/nyw091

10. Papavero L, Thiel M, Fritzsche E, Kunze C, Westphal M, Kothe R. Lumbar spinal stenosis: prognostic factors for bilateral microsurgical decompression using a unilateral approach. Neurosurgery 2009;65(6 suppl):182-7. https://doi.org/10.1227/01.NEU.0000341906.65696.08

11. Mayer HM, Microsurgical decompression of acquired (degenerative) central and lateral spinal canal stenosis. En: Mayer HM (ed.). Minimally invasive spine surgery. Berlin, Germany: Springer; 2000:105-16.

12. LaRocca H, Macnab I. The laminectomy membrane: studies in its evolution, characteristics, effects and prophylaxis in dogs. J Bone Joint Surg Br 1974:56(3):545-50. https://doi.org/10.1302/0301-620X.56B3.545

13. Raffo CS, Lauerman WC. Predicting morbidity and mortality of lumbar spine arthrodesis in patients in their ninth decade. Spine (Phila Pa 1976) 2006;31(1):99- 103. https://doi.org/10.1097/01.brs.0000192678.25586.e5

14. Boukebir MA, Berlin CD, Navarro-Ramirez R, Heiland T, Schöller K, Rawanduy C, et al. Ten-step minimally invasive spine lumbar decompression and dural repair through tubular retractors. Oper Neurosurg (Hagerstown) 2017;13(2):232-45. https://doi.org/10.1227/NEU.0000000000001407

15. Hall S, Bartleson JD, Onofrio BM, Baker HL Jr., Okazaki H, O’Duff JD. Lumbar spinal stenosis: clinical features, diagnostic procedures, and results of surgical treatment in 68 patients. Ann Int Med 1985;103(2);271-5. https://doi.org/10.7326/0003-4819-103-2-271

16. Usman M, Ali M, Khanzada K, Ishaq M, Naeem-ul-Haq, Aman R, et al. Unilateral approach for bilateral decompression of lumbar spinal stenosis: a minimal invasive surgery. J Coll Physicians Surg Pak 2013;23(12):8526. PMID: 24304987 
17. Arai Y, Hirai T, Yoshii T, et al. A prospective comparative study of 2 minimally invasive decompression procedures for lumbar spinal canal stenosis: unilateral laminotomy for bilateral decompression (ULBD) versus musclepreserving interlaminar decompression (MILD). Spine 2014;39(4):332-40. https://doi.org/10.1097/BRS.0000000000000136

18. Wipplinger C, Kim E, Lener S, Navarro-Ramirez R, Kirnaz S, Hernandez RN, et al. Tandem microscopic slalom technique: the use of 2 microscopes simultaneously performing unilateral laminotomy for bilateral decompression in multilevel lumbar spinal stenosis. Global Spine J 2020;10(2_suppl):88S-93S. https://doi.org/10.1177/2192568219871918

19. Parikh K, Tomasino A, Knopman J, Boockvar J, Härtl R. Operative results and learning curve: microscope-assisted tubular microsurgery for 1- and 2-level discectomies and laminectomies. Neurosurg Focus 2008;25(2):E14. https://doi.org/10.3171/FOC/2008/25/8/E14 